\title{
Postshock learning and conditioned defensive burying
}

\author{
JOHN P. J. PINEL, MICHAEL J. MANA, and DONALD M. WILKIE \\ University of British Columbia, Vancouver, British Columbia, Canada
}

\begin{abstract}
Natural sources of aversive stimuli are frequently well-defined material objects that are present both before and after the aversive event. In the present experiment, rats acquired information about such a source after the aversive event and used this information to guide their subsequent defensive reactions to it. The rats were shocked by one of two possible sources, either a black or a striped prod, mounted on opposite end walls of the test chamber. Immediately following the shock, the houselights were momentarily extinguished and the patterns on the two prods were automatically switched for subjects in the experimental condition or left unchanged for subjects in the control condition. The rats were left in the chamber for another 5 min with the patterns in their new positions before being removed for 2 min while the two prods were mounted on the side walls. During the ensuing test of conditioned defensive burying, the rats in the control condition directed the majority of their burying behavior at the prod exhibiting the pattern displayed by the shock source prior to and during the shock administration. In contrast, the rats in the experimental group buried the prod exhibiting the pattern displayed by the shock source during the postshock period more than they did the prod displaying the pattern present on the shock source prior to and during the shock administration.
\end{abstract}

The conditional stimuli in most aversive conditioning experiments have two important properties. They are ethereal; that is, they are stimuli such as lights and tones that lack physical substance. And they are not spatially contiguous with the unconditional stimulus; that is, they are not the source of the aversive stimulation, which is usually a diffuse shock administered through the floor of the test chamber. Although the neutrality and controllability of such stimuli have been employed to good advantage in numerous studies of aversive conditioning, their predominance may have excluded from widespread consideration important issues concerning aversive conditioning and its role in the survival of animals in the wild. The focus of the present experiment is on one such issue.

This experiment grew out of an interest in the behavior displayed by rats in those situations in which an aversive stimulus is delivered from a clearly defined, material, inanimate source. In addition to the well-documented conditioned defensive burying observed following such an aversive experience (see reviews by Pinel \& Treit, 1983, and Pinel \& Wilkie, 1983), most rats direct numerous stereotypical approach sequences at the source (Pinel, Hoyer, \& Terlecki, 1980). Each of these approach sequences begins with the subject moving cautiously toward the source of aversive stimulation from a distant part of the chamber. As the rat nears the source, its hindquarters stop while its front legs and head slowly continue, thus leaving the rat in an extended position. The rat then leans forward, frequently sniffing and, if close enough, con-

Supported by the Natural Sciences and Engineering Research Council of Canada. Thanks are extended to W. J. Jacobs for his helpful comments. The authors' mailing address is: Department of Psychology, University of British Columbia, Vancouver, B.C., Canada V6T 1 Y7. tacting the source with its vibrissae. This posture of "stretched attention" (Grant \& Mackintosh, 1963; Van der Poel, 1979) typically persists for a few seconds until the subject quickly backpedals while maintaining its orientation toward the source.

Hudson (1950) hypothesized that one function of the approach behavior that follows an aversive event might be to facilitate the subsequent identification of the offending object. A rat unexpectedly hurt by an inanimate object might, after a reflexive withdrawal, cautiously approach the object to gain further information about it. In most situations, the occurrence of this postshock learning would be indistinguishable from any learning that had occurred prior to or during the occurrence of the aversive stimulus. However, in the present experiment, certain features of the shock source were changed during a brief period of darkness immediately following the shock so that the capacity of preshock and postshock cues to elicit defensive behavior could be compared. The present experiment supports Hudson's hypothesis by showing that rats direct their subsequent conditioned defensive burying at a prod displaying cues that were not present on the shock source until after the shock.

\section{METHOD}

The methods were adaptations of those employed in previous studies of discriminated defensive burying (e.g., Pinel et al., 1980; Terlecki, Pinel, \& Treit, 1979). The subjects were 43 naive male $300-$ to $350-\mathrm{g}$ hooded rats (Charles River Canada, St. Constant, Quebec). They were housed in groups of 5 or 6 in wire-mesh cages. The colony rooms were maintained on a 12-h light/dark cycle, and all phases of the experiment were conducted during the light phase. Purina Lab Chow and water were continuously available in the home cages. 
The rats were placed in a $45 \times 40 \times 40 \mathrm{~cm}(1 \times \mathrm{w} \times \mathrm{h})$ opaque Plexiglas test chamber in groups of 5 or 6 for 30 -min periods on each of 4 consecutive habituation days. During half of each 30min habituation period, the floor of the chamber was a layer of commercial ground corncob bedding material (San-i-cel) leveled to a height of $5 \mathrm{~cm}$; during the other half-order counterbalanced-the bedding was covered with a sheet of transparent Plexiglas.

Before the conditioning trial on Day 5 , two prods were mounted in the test chamber, each through holes in the center of the end walls $2 \mathrm{~cm}$ above the level of the bedding material. Four different prods were used in the experiment. Each prod consisted of a wooden dowel, $6 \mathrm{~cm}$ long, inside a transparent vinyl tubing (external diameter $=15 \mathrm{~mm}$ ), which was wrapped with a pair of uninsulated wires. The wooden cores of two of the prods used in the experiment were painted black; the cores of the other two were painted in black and white stripes. Between the painted wooden dowels and the overlying transparent wire-wrapped vinyl tubing was a thin retractable plastic tube. When this retractable core cover was in place, it was clearly visible through the transparent vinyl, and it effectively concealed the painted wooden core. The retractable core covers on two of the prods were painted black; the other two were painted in black and white stripes. Accordingly, the four different prods used in the experiment were: black core/striped cover; striped core/black cover; black core/black cover; and striped core/striped cover. Figure 1 is a drawing of one of the prods (black core/striped cover) with the plastic cover partially retracted to better reveal the structure of the prod.

During the conditioning trial, the core covers of all of the prods were in place, and the pattern of the prod was thus the pattern of the cover during this stage of the experiment. At the beginning of the conditioning trial, all subjects were ostensibly exposed to the same prods, a black prod mounted on one end wall and a striped prod mounted on the other. Prior to the Day 5 conditioning trial, the black prod was randomly designated as the shock prod for half the rats $(n=22)$ and the striped prod was designated as the shock prod for the other half $(n=21)$. During a conditioning trial, the bedding in the test chamber was covered with the Plexiglas sheet to prevent the confounding effects of any conditioned defensive burying that might have otherwise occurred during this stage of the experiment. To begin each conditioning trial, a rat was placed in the center of the test chamber, and when it first touched the designated shock prod with a forepaw, it was shocked from an 800 -V source in series with an $80-\mathrm{k} \Omega$ resistor. The shock was turned on by the

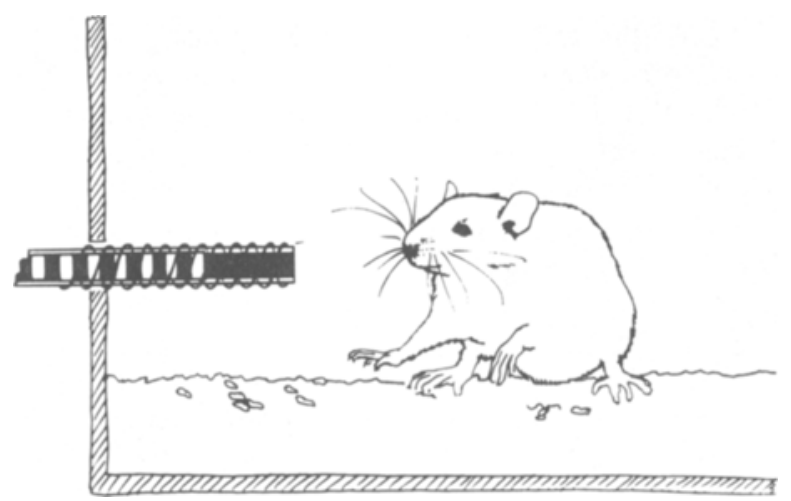

Figure 1. An illustration of the structure of one of the special prods employed in the present experiment. Represented in this illustration is a prod with a black core and a striped core cover, with its cover partially retracted to display the underlying core. During the conduct of the experiment, the test chamber lights were briefly extinguished during the retraction of the collar so that the subjects could not see the pattern switch occurring. experimenter once the rat established forepaw contact with the prod; it was terminated by the withdrawal of the rat. This procedure produces shocks averaging $7.9 \mathrm{~mA}$ and $45.2 \mathrm{msec}$.

Once the shock was administered, the lights in the test room were extinguished for $2 \mathrm{sec}$ while the retractable core covers were automatically retracted from the two prods, thereby exposing the underlying black or striped wooden cores. There were two main conditions. In the same condition $(n=23)$, the patterns of the wooden cores were the same as those present on the prods prior to and during the shock administration. In the switch condition $(n=20)$, the patterns of the exposed cores were opposite to those present during the preshock period. Thus, during the ensuing 5-min postshock exposure period, rats in the same condition were confronted with prods that appeared identical to those present before the delivery of the shock. In contrast, for those in the switch condition, the striped prod was ostensibly where the black prod had been and vice versa.

Immediately after the 5-min postshock exposure period, the rats were removed from the test chamber for $2 \mathrm{~min}$. During this time, the Plexiglas sheet covering the bedding material was removed and the two prods were moved from the end walls to the side walls of the test chamber so that the subjects could not use position as a cue to guide subsequent interactions with the prods. The subjects were then placed back in the test chamber, and their behavior was observed for a 15-min period. The hypothesis was that the appearance of the shock and control prods during the 5-min postshock exposure period would influence the direction of the conditioned defensive burying observed during the 15-min test, or more specifically, that rats in the switch condition would be less likely to selectively bury the prod pattern present prior to shock delivery than would rats in the same condition.

\section{RESULTS}

It is readily apparent in Figure 2 that the results were in accord with the experimental hypothesis; switching the pattern associated with the shock prod before the postshock observation period had a substantial effect on subsequent burying behavior. An overall analysis of variance revealed a significant conditions effect $[F(1,41)=38.1$, $p<.0001]$ and a significant condition $\times$ prod interaction $[F(1,41)=13.75, p<.007]$. Subsequent post hoc analysis of the conditions effect revealed that the rats in the switch condition spent more time burying than the subjects in the same condition (Newman-Keuls $p<.05$ ). Post hoc analysis of the interaction revealed that the rats in the same condition spent more time burying the prod displaying the pattern associated with the delivery of the shock than they did the control prod (Newman-Keuls $p<.05)$. In contrast, the rats in the switch condition, rats for which the patterns on the shock and control prods were switched following the delivery of shock, buried the prod that displayed the pattern present on the shock prod in the postshock period more than they did the prod displaying the pattern present on the shock prod during the preshock period (Newman-Keuls $p<.05$ ).

\section{DISCUSSION}

The results support Hudson's (1950) hypothesis that postshock learning plays an important role in the defensive behavior of rats. The rats in the condition in which the patterns displayed by the shock and control prods were 


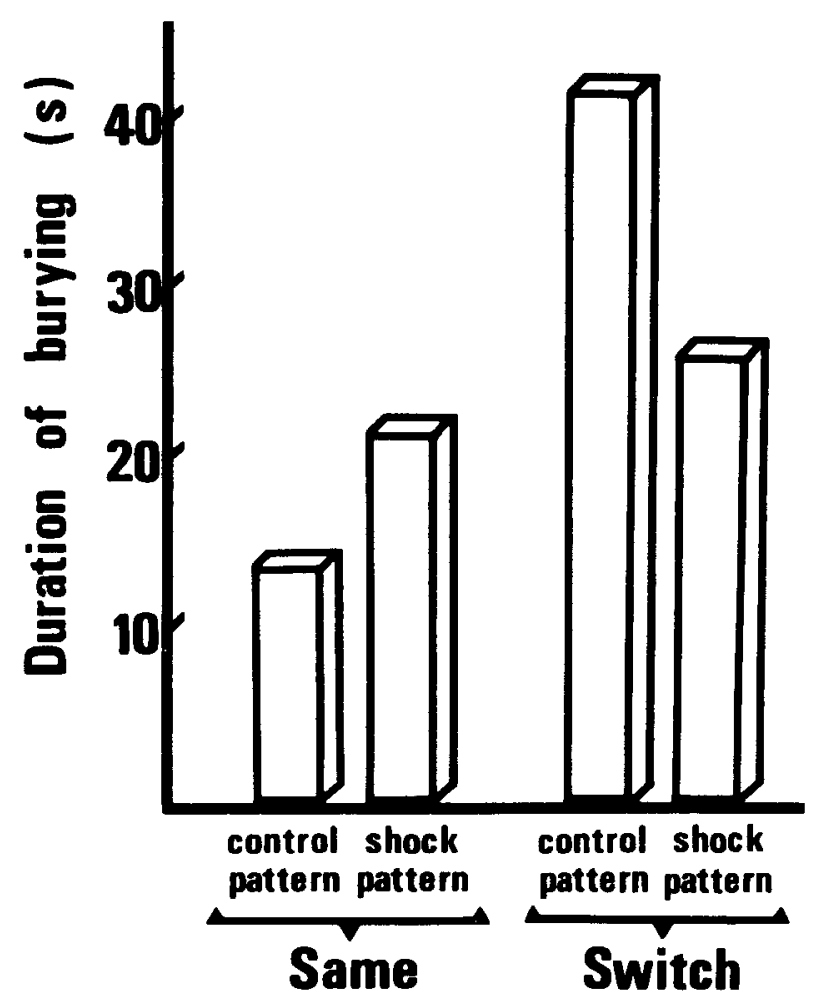

Figure 2. Mean duration of burying as a function of experimental condition. For the switch subjects, the striped and black patterns on the shock and control prods were ostensibly switched during the extinction of the test chamber lights immediately after the shock. For the same subjects, the patterns on the shock and control prods were the same after the shock as they had been before. All subjects remained in the chamber with the prods for 5 min following the shock. This figure displays the amount of time that the same and switch subjects spent burying the pattern that had delivered the shock as opposed to the control pattern during a subsequent 15 -min test.

switched following the shock spent less time burying the prod displaying the pattern present on the shock source prior to delivery of the shock than they did burying the prod displaying the pattern present after shock. Thus, this experiment demonstrates that rats do, in fact, learn about sources of aversive stimuli after their presentation and use this information to direct subsequent defensive behavior.

It is interesting to note that in the switch condition the rats actually spent more time burying the prod displaying the postshock pattern than they did burying the prod displaying the preshock pattern. This finding confirms the reports of Keith-Lucas and Guttman (1975), who found that rats avoided stimuli associated with shock after its delivery more than they did stimuli presented just before the delivery. Spetch, Terlecki, Pinel, Wilkie, and Treit (1982) found that the defensive burying conditioned in rats by a multiple-trial backward conditioning procedure was as robust as that produced by forward conditioning. In view of the above findings, it would seem a mistake to focus the experimental investigation of avoidance conditioning on prereinforcement information processing to the exclusion of postreinforcement effects.
Bolles and Fanselow (1980) and Crawford and Masterson (1982) have suggested that the investigatory behavior that animals engage in following an aversive event can provide information that influences the subsequent selection of an appropriate defensive response. An animal capable of learning about the source of an aversive stimulus subsequent to its occurrence would have an obvious survival advantage over an animal unable to do so (see, e.g., Keith-Lucas \& Guttman, 1975; Spetch et al., 1982). Accordingly, it is not surprising that rats, and presumably other animals, have the capacity for learning about the sources of aversive stimuli after their administration. What is surprising is that the phenomenon has not been more thoroughly investigated. However, an examination of the conditional cues employed in most traditional aversive conditioning paradigms suggests an explanation. In many instances, these cues are stimuli such as lights or tones that are temporally contingent with the aversive stimulus but lack physical substance and spatial contiguity with the source of aversive stimulation. Perhaps the lack of substance and/or the spatial separation from the aversive stimulus that characterizes this type of conditional stimuli make it difficult for an animal to direct its exploratory behavior at them following an aversive event. Although considerable evidence for backward conditioning has come from studies in which such ethereal stimuli have served as the conditional stimuli (see Spetch, Wilkie, \& Pinel, 1981 for a review), it is our view that the controversy that has surrounded the study of backward conditioning (see Hall, 1984) is in part due to the use of less than optimal conditions to demonstrate its existence.

Mackintosh (1983) recently provided experimental support for the idea that spatial contiguity between the CS and UCS is an important factor in avoidance conditioning. Rats shocked through a prod when it was contacted demonstrated substantial avoidance conditioning in only one trial; in contrast, rats shocked through a grid floor when the prod was contacted did not. Similarly, Rescorla and Cunningham (1979) found that spatial contiguity between first- and second-order conditional stimuli promotes better acquisition in a second-order conditioning paradigm. Rescorla (1980) has suggested that the potent role in conditioning for spatial contiguity between stimuli should not be surprising: "In most naturally occurring sequences, signals bear a physical relation to what they signal and consequently often emanate from the same place"' (pp. 54-55).

An aspect of the results that we did not anticipate was the significantly greater amount of burying displayed by the rats in the switch condition (see Figure 2). Why should the rats in this condition-where the shock prod displayed one pattern during the preshock period and another in the postshock period-engage in more burying than the rats for which the preshock and postshock patterns were unchanged? We believe that the answer may lie in an analysis of the subjects' perception of the experimental manipulation. In our view, switching the patterns on the prods may have done more than simply present the sub- 
jects with a new array of stimuli; it may have presented them with a perceptual experience unlike any that they had previously encountered, the sudden transformation of one object to another. This provocative aspect of the experimental treatment may have increased the overall aversiveness of the situation, thus accounting for the elevated levels of burying in the switch condition.

The burgeoning interest in forms of animal learning that mimic situations that occur in animals' natural environments may stimulate interest in the role of information obtained after the occurrence of biologically important events. Interesting lines of recent research illustrate this point and suggest possibilities for future study. First, Sherry, Krebs, and Cowie (1981) have suggested that the investigatory behavior occurring after the establishment of a cache site might help the marsh tit recover cached food. After hiding a seed, the marsh tit stereotypically inspects the cache site with each eye in rapid succession. Sherry et al. have suggested that these visual fixations might help the marsh tit subsequently recognize its cache sites. Second, Sutherland and Dyck (1984) have suggested that similar exploratory behavior might play an important role in the learning of the Morris water maze by rats (Morris, 1981). In the Morris water maze, rats must learn to find a platform hidden just under the surface of a "milky" water bath in order to climb from the water. During early trials, rats spend considerable time rearing and turning once they have reached the safety of the platform. Sutherland and Lingaard (1982) have suggested that this behavior reflects an active processing of visual cues that are subsequently used to find the platform.

The evidence accumulated by nearly a century of research based upon traditional learning paradigms presents a formidable case for the importance of information occurring prior to reinforcement in the acquisition of learned associations. However, the present data, as well as other recent research (e.g., Meck, 1985), suggest that the preoccupation with such information processing may have excluded from widespread consideration the learning that takes place after reinforcement and its important role in directing subsequent behavior.

\section{REFERENCES}

Bolles, R. C., \& Fanselow, M. S. (1980). A perceptual-defensiverecuperative model of fear and pain. Behavioral \& Brain Sciences, 3, 291-323.

Crawford, M., \& Masterson, F. A. (1982). Species-specific defense reactions and avoidance learning. Pavlovian Journal of Biological Science, 17, 204-214.

Grant, E. C., \& Mackintosh, J. H. (1963). A comparison of the social postures of some common laboratory rodents. Behaviour, 21, 246-259.

HaLl, J. F. (1984). Backward conditioning in Pavlovian type studies. Pavlovian Journal of Biological Science, 19, 163-168.

Hudson, B. B. (1950). One-trial learning in the domestic rat. Genetic Psychology Monographs, 41, 99-145.

Keith-Lucas, T, , \& Guttman, N. (1975). Robust single-trial delayed backward-conditioning. Joumal of Comparative \& Physiological Psychology, 88, 468-476.

MACKINTOSH, N. J. (1983). Conditioning and associative learning. Oxford: Oxford University Press.

MECK, W. H. (1985). Postreinforcement signal processing. Journal of Experimental Psychology: Animal Behavior Processes, 11, 52-70.

MorRIs, R. G. M. (1981). Spatial localization does not require the presence of local cues. Learning \& Motivation, 12, 239-260.

Pinel, J. P. J., Hoyer, E., \& Terlecki, L. J. (1980). Defensive burying and approach-avoidance behavior in the rat. Bulletin of the Psychonomic Society, 16, 349-352.

Pinel, J. P. J., \& Treit, D. (1983). The conditioned defensive burying paradigm and behavioral neuroscience. In T. E. Robinson (Ed.), Behavioral approaches in brain research (pp. 212-234). Oxford: Oxford University Press.

Pinel, J. P. J., \& Wilkie, D. M. (1983). Conditioned defensive burying: $A$ biological and cognitive approach to avoidance learning. In R. L. Mellgren (Ed.), Animal cognition and behavior (pp. 285-318). Amsterdam: North-Holland.

Rescorla, R. A. (1980). Pavlovian second-order conditioning. Hillsdale, NJ: Erlbaum.

Rescorla, R. A., \& CunNingham, C. L. (1979). Spatial contiguity facilitates Pavlovian second-order conditioning. Journal of Experimental Psychology: Animal Behavior Processes, 5, 152-161.

SherRY, D. F., Krebs, J. R., \& Cowie, R. J. (1981). Memory for the location of stored food in marsh tits. Animal Behaviour, 29, 1260-1266.

SPetch, M. L., Terlecki, L. J., Pinel, J. P. J., Wilkie, D. M., \& TREIT, D. (1982). Excitatory backward conditioning of defensive burying in the rat. Bulletin of the Psychonomic Society, 19, 111-114.

Spetch, M. L., Wilkie, D. M., \& Pinel, J. P. J. (1981). Backward conditioning: A re-evaluation of the empirical evidence. Psychological Bulletin, 89, 167-175.

SUTHERLAND, R. J., \& DYCK, R. H. (1984). Place navigation by rats in a swimming pool. Canadian Journal of Psychology, 38, 322-347.

Sutherland, R. J., \& LingaARd, R. C. (1982). Being there: A novel demonstration of latent spatial learning in the rat. Behavioral \& Neural Biology, 36, 103-107.

Terlecki, L. J., Pinel, J. P. J., \& Treit, D. (1979). Conditioned and unconditioned defensive burying in the rat. Learning \& Motivation, 10, 337-350.

VAN DER POEL, A. M. (1979). A note on 'stretched attention', a behavioural element indicative of an approach-avoidance conflict in rats. Animal Behaviour, 27, 446-450.

(Manuscript received October 15, 1985; revision accepted for publication April 15, 1986.) 